\title{
Essais
}

ESSAIS

Revue interdisciplinaire d'Humanités

\section{Les lieux historiques et leur dimension pédagogique. Le débat allemand contemporain}

\section{Christine Pflüger}

\section{(2) OpenEdition \\ 1 Journals}

Édition électronique

URL : http://journals.openedition.org/essais/5548

DOI : $10.4000 /$ essais. 5548

ISSN : 2276-0970

Éditeur

École doctorale Montaigne Humanités

\section{Édition imprimée}

Date de publication : 15 janvier 2015

Pagination : 18-36

ISBN : 978-2-9544269-4-5

ISSN : 2417-4211

\section{Référence électronique}

Christine Pflüger, « Les lieux historiques et leur dimension pédagogique. Le débat allemand contemporain », Essais [En ligne], 6 | 2015, mis en ligne le 30 novembre 2020, consulté le 02 décembre 2020. URL : http://journals.openedition.org/essais/5548; DOI : https://doi.org/10.4000/essais.5548 


\section{Les lieux historiques et leur dimension pédagogique. Le débat allemand contemporain}

\section{Christine Pflüger}

" Si j'ai bien compris, un lieu historique, c'est un endroit qui ne change pas de place...»-c'est ainsi qu'un étudiant a résumé la définition lors d'un cours sur la dimension didactique des lieux historiques. En effet, en faisant la différence entre les lieux d' "apprentissage de compétences historiennes " - comme les archives, les musées, etc. - et les "lieux historiques", les principaux manuels de didactique de l'histoire définissent ces derniers comme des sites authentiques, restés dans leur «cadre naturel», leur " environnement originel $»^{1}$, ce qui en fait des lieux spécifiques.

Afin d'éviter toute confusion, il faut souligner la différence entre le concept didactique des "lieux historiques ", d'un côté, et le concept historiographique des «lieux de mémoire " selon Pierre Nora de l'autre. ${ }^{2}$ Tandis que ce dernier implique non seulement des lieux historiques au sens propre, c'est-à-dire des endroits précis, mais aussi des « lieux » au sens figuré, métaphorique et immatériel, comme des dates (le 14 juillet par exemple), des événements ainsi que des personnages - réels ou mythiques - ayant marqué l'histoire de la France,

1 Ulrich Mayer, "Historische Orte als Lernorte », in Ulrich Mayer, Hans-Jürgen Pandel et Gerhard Schneider (éd.), Handbuch Methoden im Geschichtsunterricht, Schwalbach/Ts., Wochenschau Verlag, Forum HistorischesLernen, 2004, p. 389-407, icip. 390; Waltraud Schreiber, "Historische Stätten », in Waltraud Schreiber (éd.), Erste Begegnungen mit Geschichte. Grundlagen historischen Lernens I, Neuried, Ars una, Bayerische Studien zur Geschichtsdidaktik 1, 1999, p. 589-606 ; Christian Kuchler, Historische Orte im Geschichtsunterricht, Schwalbach/Ts., Wochenschau Verlag, 2012 (Methoden historischen Lernens).

2 Pierre Nora (éd.), Les Lieux de mémoire, 7 vol., Paris, 1984-1993. Le concept de Pierre Nora a été transposé Outre-Rhin dans le cadre d'un projet mené sous la direction des historiens Étienne François et Hagen Schulze qui ont dirigé une sélection d'études tout en tenant compte des problématiques et spécificités de la mémoire de l'histoire allemande en Allemagne, ainsi que de la mémoire « double » marquée par les quarante ans d'existence de deux États allemands, la RFA et la RDA. Étienne François et Hagen Schulze (éd.), Deutsche Erinnerungsorte. 3 vol., Frankfurt/M. et al., 2001. La version traduite en français est plus succinte : Étienne François et Hagen Schulze (éd.), Mémoires allemandes, Paris, Gallimard, 2007, 800 p. 
des notions, des œuvres littéraires ou musicales, des traditions populaires et des pratiques culturelles constituant l'inventaire symbolique et collectif qui, selon P. Nora, représente le fondement d'une perception de l'histoire de la France, le concept des "lieux historiques", tel qu'il est discuté au sein de la recherche allemande en didactique de l'histoire, se réfère en revanche uniquement à des lieux concrets.

Selon le didacticien allemand Ulrich Mayer, un lieu historique représente une réalité stable, immobile, ${ }^{3}$ ce qui ne veut pas dire que le site même ne change pas au cours des temps. Par "lieu historique ", U. Mayer entend tout endroit où un évènement " historique " a eu lieu, tout endroit représentant le cadre d'une évolution historique ou qui fut un cadre de vie à une certaine époque. ${ }^{4}$ Cette notion de lieu historique couvre donc une multitude d'endroits divers - à commencer par toutes sortes de châteaux, les anciennes cachettes des camisards dans les Cévennes ou des maquis en Savoie, les installations de la première ligne " télégraphique " de Claude Chappe entre Paris et Lille, en passant par les places de marché, des anciens lavoirs ou même un four à chaux en pleine campagne. Ainsi, on compte parmi les lieux historiques non seulement les sites connus par les touristes, mais également des ruines - même des usines désaffectées - envahies par les broussailles, des lieux où un bâtiment a été détruit ou au contraire élargi et réamenagé, une ancienne chaussée recouverte d'herbes et d'autres endroits semblables, traces d'époques lointaines.

Le débat allemand sur la "valeur pédagogique " de ces lieux historiques a plusieurs dimensions, la première concerne la pédagogie de l'enseignement scolaire de l'histoire. ${ }^{5}$ Celle-ci fait bien sûr partie intégrante de la dimension didactique ; cependant, comme la dimension didactique couvre aussi des aspects plus théoriques, on les traitera ici comme deux volets du débat. La dimension didactique sera donc présentée dans le cadre d'une approche analytique. Quant aux lieux historiques, la didactique de l'histoire qui se penche sur les modes de transmission de l'histoire liés aux lieux a pour objectif l'analyse des représentations du passé sous-jacentes aux agencements des sites. Elle s'intéresse ainsi aux constructions de sens historique lors des aménagements et agencements des sites, mais elle porte aussi son attention aux constructions de

3 Ulrich Mayer, op. cit., p. 390.

4 Ibid., p. 390.

5 Outre l'article d'Ulrich Mayer cf. également Hilke Günther-Arndt, " Erkundender Geschichtsunterricht ", in Hilke Günther-Arndt (éd.), Geschichtsmethodik. Handbuch für die Sekundarstufe I und II, Berlin, Cornelsen Scriptor, 2007, p. 119 ; Berit Pleitner, "Lokalerkundung ", in Hilke Günther-Arndt (éd.), op. cit., p. 138-141 ; Edda Grafe, "Exkursion ", in Hilke Günther-Arndt (éd.), op. cit., p. 134-137 ; Meik Zülsdorf-Kersting, "Gedenkstättenarbeit ", in Hilke Günther-Arndt (éd.), op. cit., p. 142-147. À propos des lieux d'apprentissage de compétences historiennes cf. par exemple les contributions dans l'ouvrage collectif de Saskia Handro et Bernd Schönemann (éd.), Orte historischen Lernens, LIT Verlag, Berlin, 2008 (Zeitgeschichte - Zeitverständnis Band 18). 
sens et aux représentations que se font les visiteurs. La troisième dimension est la dimension politique, sociale et culturelle qui touche les questions d'aménagement d'un site telles qu'elles sont discutées par les conseils municipaux mais aussi dans l'espace public, qu'il s'agisse d'un lieu de commémoration, d'un bâtiment à entretenir ou de tout autre lieu historique. Enfin, le quatrième aspect du débat concerne le travail pédagogique sur les lieux de commémoration; il s'agit là d'une question très spécifique.

Avant de présenter les quatres volets du débat, passons brièvement en revue les principales étapes de la genèse de la question des "lieux historiques " dans le cadre de l'Allemagne post-1945. Enseigner l'histoire régionale en quittant la salle de classe pour visiter avec les élèves des sites historiques ou des musées n'est pas une idée nouvelle dans l'école de l'après-guerre. Le concept pédagogique des excursions se trouvait déjà dans des publications du début du $\mathrm{XX}^{\mathrm{e}}$ siècle. ${ }^{6}$ Néanmoins, il faut attendre les années soixante-dix pour voir se relancer le débat pédagogique concernant l'enseignement de l'histoire sur des sites historiques, ${ }^{7}$ qui conduit à la visite d'endroits authentiques et à l'interprétation des traces historiques inscrites dans les villes et les paysages. Ce débat fut fortement influencé par l'approche scientifique de "l'histoire orale " (oral history), appliquée non seulement en recherche historique mais aussi au sein des " ateliers historiques " régionaux fréquentés par un public plus large. ${ }^{8}$ Bernd Hey fut l'un des auteurs auquels on doit beaucoup d'impulsions novatrices quant aux projets liés à l'enseignement - scolaire et extra-scolaire - de l'histoire. ${ }^{9}$ Il mit

6 Cf. par exemple August Tecklenburg, Bildender Geschichtsunterricht. Der Geschichtsunterricht in der Grundschule. Erster selbständiger Geschichtsunterricht aufheimatlicher Grundlage in Begründung und Beispiel, $2^{\mathrm{e}}$ édition, Hannover, 1921 ( $1^{\mathrm{re}}$ édition 1904); A. Stocker, Unterrichtsplan für die Volksschule vom 12. April 1924 mit Erläuterungen und methodischen Bemerkungen, Verlag der Konkordia A.G., Bühl (Baden), 1924, p. 43. On ne retracera pas ici la genèse du concept pédagogique de l'enseignement par le vécu qui remonte encore beaucoup plus loin.

7 On parle ici du débat dans le cadre de l'enseignement scolaire de l'histoire et dans le cadre de la formation universitaire des enseignants en RFA. Quant au développement des concepts pédagogiques et didactiques en RDA le lecteur pourra se référer par exemple à Andreas Fischer, Das Bildungssystem der DDR, Darmstadt, 1992, ou à Christoph Führ et Carl-Ludwig Furck (éd.), Handbuch der deutschen Schulgeschichte, vol. IV: 1945 bis zur Gegenwart, t. II : Deutsche Demokratische Republik, München, 1998. Hans-Dieter Schmid a publié en RFA une analyse de l'enseignement de l'histoire en RDA : Hans-Dieter Schmid, Geschichtsunterricht in der DDR. Eine Einführung, Ernst Klett Verlag, Stuttgart, 1979 (Anmerkungen und Argumente zur historischen und politischen Bildung ; 25). On trouve une documentation sur le développement des conceptions éducatives en zone d'occupation soviétique pendant l'après-guerre chez Siegfried Baske et Matha Engelbert, Dokumente zur Bildungspolitik in der sowjetischen Besatzungszone, Bonn-Berlin, 1966.

8 Cf. Ulrich Mayer, op. cit., p.389.

9 Cf. par exemple Bernd Hey, Die historische Exkursion. Zur Didaktik und Methodik des Besuchs historischer Stätten, Museen und Archive, Stuttgart 1978 ; Bernd Hey, «Archive und historische Stätten als außerschulische Lernorte. Zum Begriff der historischen Exkursion ", in Geschichte in Wissenschaft und Unterricht 31 (1980), p. 30-40. 
l'accent sur l'acquisition de connaissances historiques par le biais du travail sur les lieux, sur des documents et des objets authentiques, bref, sur l'acquisition de connaissances par des recherches sur place, dans les archives et les musées. Le débat didactique a pris un tour encore plus différencié depuis les années 1990, quand la différenciation s'est établie de plus en plus nettement entre les lieux historiques d'un côté, et les lieux d'apprentissage et d'acquisition de compétences historiennes, comme les archives et les bibliothèques ainsi que les lieux de représentation de l'histoire tels les musées, de l'autre. ${ }^{10}$

Une autre strate s'est ajoutée au débat concernant tout d'abord la gestion et l'agencement des anciens lieux de la terreur du régime national-socialiste, ${ }^{11}$ puis une autre, dans l'Allemagne réunifiée, portant sur les anciens camps de concentration nazis qui avaient à nouveau servi comme " camps spéciaux " d'internement du temps de l'URSS et de la RDA. Au printemps 1990, une discussion publique très controversée s'est engagée quant à l'agencement et l'aménagement de ces camps comme lieux de commémoration. ${ }^{12}$ En 1992, la Commission d'enquête Aufarbeitung von Geschichte und Folgen der SED-Diktatur in Deutschland [Travail critique sur l'histoire et les conséquences de la dictature du SED en Allemagne] fut mise en place par le gouvernement allemand, suivie en 1995-1998 par la Commission Überwindung der Folgen der SED-Diktatur im Prozeß der deutschen Einheit [Gestion critique des conséquences de la dictature du SED dans le processus de l'unité allemande]. ${ }^{13}$

10 Cf. Ulrich Mayer, op. cit., p. 389.

11 Cf. par exemple Stefanie Endlich, "Orte des Erinnerns - Mahnmale und Gedenkstätten », in Peter Reichel, Harald Schmid et Peter Steinbach (éd.), Der Nationalsozialismus - Die zweite Geschichte. Überwindung - Deutung - Erinnerung, Lizenzausgabe für die Bundeszentrale für politische Bildung, Bonn, 2009, p. 350-377 (Verlag Beck, München, 2009).

12 Cf. Bodo Ritscher, "Die wissenschaftliche Aufarbeitung der Geschichte der sowjetischen Speziallager in der SBZ/DDR seit Beginn der 1990er Jahre - Zwischenbilanz und Ausblick ", in PetraHaustein, Annette Kaminsky, Volkhard Knigge et Bodo Ritscher (éd.), Instrumentalisierung, Verdrängung, Aufarbeitung. Die sowjetischen Speziallager in der gesellschaftlichen Wahrnehmung 1945 bis heute, Wallstein Verlag, Göttingen 2006, p. 170-192 ; Petra Haustein, " Geschichte im Dissens. Die Auseinandersetzungen um die Gedenkstätte Sachsenhausen nach dem Ende der DDR ", in Petra Haustein et al. (éd.), op. cit., p. 133-148 ; Gudrun Schwarz, "Zur Gedenkstätte Ravensbrück », in Jürgen Danyel (éd.), Die geteilte Vergangenheit. Zum Umgang mit Nationalsozialismus und Widerstand in beiden deutschen Staaten. Akademie Verlag Berlin, 1995 (Zeithistorische Studien Band 4), p. 153-162 ; Bodo Ritscher, "Die NKWD/ MWD-Speziallager » in Deutschland. "Anmerkungen zu einem Forschungsgegenstand ", in Jürgen Danyel (éd.), op. cit., p. 163-179. Quant au débat sur les camps dans l'immédiat aprèsguerre, cf. Wolfram von Scheliha, "Die sowjetischen Speziallager - ein Symbol des kommunistischen Unrechts in der publizistischen Auseinandersetzung zwischen Ost und West bis zum Bau der Berliner Mauer 1961 ", in Petra Haustein et al. (éd.), op. cit., p. 10-28 ; enfin, quant aux expositions installées sur le terrain des camps, cf. Annette Kaminsky, « Ausstellungen zu den sowjetischen Speziallagern in der SBZ/DDR - eine vergleichende Betrachtung ", in Petra Haustein et al. (éd.), op. cit., p. 149-169.

13 Cf. Bodo Ritscher, "Die wissenschaftliche Aufarbeitung der Geschichte der sowjetischen Speziallager in der SBZ/DDR seit Beginn der 1990er Jahre - Zwischenbilanz und Ausblick ", in Petra Haustein et al. (éd.), op. cit., p. 170-192, ici p. 176-177 ; Materialien der Enquete- 
Les deux commissions furent chargée de mener des recherches et d'effectuer des expertises sur l'histoire de ces camps; le dossier final servit de base à la "Conception des lieux de commémoration" (Gedenkstätten-Konzeption) du gouvernement allemand. ${ }^{14}$ Le travail des deux commissions fut enrichi par des projets de recherche sur ces camps spéciaux d'internement d'un côté et accompagné d'une discussion didactique et pédagogique assez vive de l'autre, concernant l'enseignement de l'histoire, l'education civique, la mémoire et la commémoration dans la société. ${ }^{15}$

\section{La dimension pédagogique}

Depuis au moins quarante ans, l'enseignement scolaire de l'histoire conçoit l'histoire en tant que discipline scientifique. ${ }^{16}$ Dans ce contexte, il est question du "potentiel didactique " ou du "potentiel pédagogique " des lieux historiques - potentiel qu'il revient aux enseignants de mettre en valeur

Kommission "Aufarbeitung von Geschichte und Folgen der SED-Diktatur in Deutschland", Baden-Baden, Frankfurt/M., 1995 ; Materialien der Enquete-Kommission "Überwindung der Folgen der SED-Diktatur im Prozeß der deutschen Einheit ", Baden-Baden, Frankfurt/M., 1999 (XVIII vol. pour les deux commissions).

14 Cf. Bodo Ritscher, "Die wissenschaftliche Aufarbeitung der Geschichte der sowjetischen Speziallager in der SBZ/DDR seit Beginn der 1990er Jahre - Zwischenbilanz und Ausblick ", in Petra Haustein et al. (éd.), op. cit., p. 170-192, ici p. 176-177; « Konzeption der künftigen Gedenkstättenförderung des Bundes und Bericht der Bundesregierung über die Beteiligung des Bundes an Gedenkstätten in der Bundesrepublik Deutschland ", in Deutscher Bundestag, Drucksache 14/1569, 27.07.1999.

15 Par exemple Norbert Frei, « Deutsche Lernprozesse. NS-Vergangenheit und Generationenfolge seit 1945 ", in Petra Haustein et al. (éd.), op. cit., p. 63-78 ; Petra Haustein, op. cit. ; Bettina Greiner, "Der Preis der Anerkennung. Zur Debatte über den Erinnerungsort der Speziallager ", in Petra Haustein et al. (éd.), op. cit., p. 114-132 ; Bernd Faulenbach, "Öffentliches Erinnern im vereinten Deutschland und in Osteuropa seit den 1990er Jahren ", in Petra Haustein et al. (éd.), op. cit., p. 233-249 ; Claudia Fröhlich, "Rückkehr zur Demokratie - Wandel der politischen Kultur in der Bundesrepublik ", in Peter Reichel et al. (éd.), op. cit., p. 105-126; Harald Schmid, "Deutungsmacht und kalendarisches Gedächtnis - die politischen Gedenktage ", in Peter Reichel et al. (éd.), op. cit., p. 175-216.

16 La période de « quarante ans " fait ici référence au discours didactique en République fédérale. Après 1968, ce dicours a connu des changements profonds touchant surtout aux approches, objectifs et méthodes de l'enseignement scolaire de l'histoire, mis en question dans le cadre des mouvements de 1968. Les années 1970 ont donc vu le développement de nouvelles approches didactiques de l'histoire ainsi qu'un rapprochement de l'enseignement de l'histoire et de l'éducation civique ou - terme suisse - de l'éducation à la citoyenneté. En RDA (jusqu'en 1989) existaient des concepts didactiques propres aux objectifs pédagogiques et au système scolaire et éducatif, qui ne pourront pas être l'objet de cet article. Les années 1990 ont vu l'essor d'études comparées des deux systèmes éducatifs et des analyses sur les façons de penser qui en résultaient chez les jeunes. Cf. par exemple Hans Süßmuth (éd.), Geschichtsunterricht im vereinten Deutschland. Auf der Suche nach einer Neuorientierung, Baden-Baden 1991, et Bodo von Borries, «Verknüpfung der Zeitebenen im Geschichtsbewusstsein? Zu Vergangenheitsdeutungen, Gegenwartswahrnehmungen und Zukunftserwartungen ost- und westdeutscher Jugendlicher, 1992 ", in Jörn Rüsen (éd.), Geschichtsbewusstsein. Psychologische Grundlagen, Entwicklungskonzepte, empirische Befunde (Beiträge zur Geschichtskultur Band 21), Köln et al., 2001, p. 281-315. 
afin de permettre aux élèves d'acquérir des connaissances historiques et des compétences historiennes, de découvrir les méthodes de la recherche scientifique et de réfléchir sur le passé à partir d'une "rencontre authentique " avec le lieu historique ${ }^{17}$. Les futurs enseignants d'histoire se familiarisent systématiquement avec ce concept lors de leur formation académique, en travaillant eux-mêmes sur des exemples régionaux.

Revenons au potentiel pédagogique des lieux historiques. La permancence et la réalité sont deux aspects importants du potentiel pédagogique des lieux historiques. ${ }^{18}$ Les visiteurs font l'expérience du réel : le lieu existe vraiment, les murs sont palpables, les traces de vie visibles, l'endroit n'est pas une fiction. S'ajoutent à ces aspects ceux de l'historicité et de l'authenticité. Évidemment, si "l'endroit ne change pas de place ", il change tout de même au cours du temps, soit en raison des intempéries naturelles, soit par des réaménagements ou agencements ou même par des destructions volontaires. Prenons comme exemples d'anciens châteaux médiévaux, tombés en ruines et souvent utilisés comme carrières pour divers chantiers au cours des siècles, puis pris en charge par le service des monuments historiques au $\mathrm{XX}^{\mathrm{e}}$ siècle dans le but de sauvegarder le patrimoine médiéval. Ces changements permettent aux élèves de réaliser la distance dans le temps. Ils s'aperçoivent alors de l'historicité des lieux, voire de l'historicité en général, de ce changement permanent auquel tout ce qui existe est soumis et qui est « le principe même de l'histoire " ${ }^{19}$. De plus, aller sur place avec des élèves au lieu de regarder des images et des photos leur permet de développer la notion de l'espace ${ }^{20}$ et d'acquérir une représentation authentique des dimensions spatiales d'un site.

À partir de l'originalité, de la visibilité et de l'évidence d'un lieu historique, c'est l'imagination du visiteur qui est stimulée. Puisqu'on ne voit jamais, à partir d'un lieu actuel, comment il était à l'origine ou à une certaine époque, il est important de dé-construire l'état actuel, de retracer l'évolution et de faire une re-construction (virtuelle) de l'état historique ainsi que de recontextualiser l'ensemble. Un exemple typique pour cette recontextualisation serait les quartiers et villages des immigrés huguenots au $\mathrm{XVII}^{\mathrm{e}}$ et $\mathrm{XVIII}{ }^{e}$ siècles dans la région de Cassel en Hesse : ce sont des villages pimpants où l'on trouve un grand nombre d'inscriptions en ancien français sur les poutres des maisons en colombages. Mais lors de l'arrivée des réfugiés à l'époque, ceux-ci étaient logés pour la plupart dans des barraques simples et provisoires avant que l'on ne construise des quartiers entiers. Il faut alors retracer ce cheminement en remontant dans le temps à partir de l'état actuel du bâtiment.

17 Il s'agit évidemment d'une « rencontre » avec un lieu dans son état actuel, présent, et en aucun cas d'une rencontre avec « le passé ». Ulrich Mayer, op. cit., p. 396-397.

18 Ulrich Mayer, op. cit., p. 392-393.

19 Ibid., p. 395.

20 Ibid., p. 393-394. U. Mayer suit ici Siegfried Münchenbach, " Geschichte vor Ort - Exkursion und Museum ", in Harald Parigger (éd.), Die Fundgrube für den Geschichts-Unterricht. Ein Nachschlagewerk für jeden Tag, Berlin, 1996, p. 315-325, ici p. 315. 
La perception d'un tel site, c'est-à-dire aussi la perception par les sens de la température, de l'air dans un bâtiment, de l'ambiance, des bruits alentour, agiront sur l'imagination du visiteur et sur la représentation qu'il se fera de l'état d'un lieu à une certaine époque en le reconstruisant mentalement et en le situant dans un contexte historique. Ce processus de dé-construction, re-construction et re-contextualisation suscite la création de sens et provoque une construction individuelle de sens historique ${ }^{21}$.

Afin que cette représentation corresponde au maximum aux résultats de la recherche scientifique actuelle, l'enseignant fournit en outre aux élèves des sources et de la documentation supplémentaires comme bases de travail, en vertu du principe didactique selon lequel tout phénomène historique doit être abordé sous des angles multiples (Multiperspektivität). ${ }^{22}$

Mises à part l'initiation à la recherche et l'acquisition de connaissances historiques, un travail "sur place » avec des élèves cherche également à éveiller l'empathie envers les victimes, envers les hommes, les femmes et les enfants qui ont vécu et souffert dans le passé. ${ }^{23}$ La capacité de se faire par exemple une idée de l'horreur et de l'enfer des camps est due au fond à une multitude de capacités comme l'empathie, l'imagination, les connaissances historiques acquises (par enseignement, les lectures, les témoignages et les récits, les films documentaires), mais aussi à la mémoire collective et culturellement transmise. ${ }^{24}$ La discussion pédagogique concernant le potentiel didactique des lieux historiques devenus lieux de commémoration - débat très spécifique sur lequel nous reviendrons plus tard - est par conséquent ciblée sur "l'éducation civique à la démocratie " ${ }^{25}$ ou, comme l'on dirait en Suisse, sur l'éducation à la citoyennetée ${ }^{26}$, qui vise à développer la conscience historique par l'apprentissage émotionnel et affectif, et la prise de conscience des valeurs démocratiques, celles d'auto-réflexion et d'autonomie. ${ }^{27}$ Selon Christian Geißler-Jagodzinski et Verena Haug, il est nécessaire de mettre en relief l'impact du passé sur le présent ainsi que la signification de l'histoire dans l'interprétation du présent et la conception du futur, afin d'aider

21 Ulrich Mayer, op. cit., p. 393-394.

22 Ibid., p. 393.

23 Christian Geißler-Jagodzinski et Verena Haug, "Gedenkstättenpädagogik - Ziele, Grenzen und Widersprüche ", in Janne Mende et Stefan Müller (éd.), Emanzipation in der politischen Bildung. Theorien - Konzepte - Möglichkeiten, Schwalbach/Taunus, Wochenschau Verlag, 2009, p. 299-329, ici p. 315-316.

24 Cf. Christian Geißler-Jagodzinski et Verena Haug, op. cit., p. 311-316, et Volkhard Knigge, Zur Zukunft der Erinnerung, Aus Politik und Zeitgeschichte (APuZ) 25-26/2010, p. 10-16, ici p. 11.

25 Ibid., p. 309. La formule employée par les auteurs est « das Lernen für Demokratie und Zivilcourage ".

26 Cf. également Volkhard Knigge, "Zur Zukunft der Erinnerung ", in Aus Politik und Zeitgeschichte (APuZ) 25-26/2010, p. 10-16, ici p. 15.

27 Christian Geißler-Jagodzinski et Verena Haug, op. cit., p. 311. On y trouvera également la littérature de référence des deux auteurs et les références bibliographiques correspondantes. 
les élèves à développer leurs capacités politiques et morales. ${ }^{28}$ Harald Welzer prône un enseignement qui s'intéresserait aux conditions sociales, politiques et socio-psychologiques du comportement humain. ${ }^{29}$

Parmi les travaux pratiques proposés aux élèves, on trouve des projets dont la méthode s'inspire de la démarche scientifique, adaptée aux conditions de l'enseignement scolaire : une investigation menée par les élèves, des recherches sur place, l'établissement d'un " état de la recherche " suivi par la présentation structurée des observations. Les élèves notent les questions qu'ils se posent et émettent ensuite des hypothèses qu'ils essayent de vérifier à partir d'une documentation supplémentaire - ainsi, ils font eux-mêmes un travail analytique d'historien. Ils découvrent la topologie sociale, politique, économique et culturelle d'un lieu à une certaine époque. S'ensuit, éventuellement, la reconstruction des événements sur le site historique. La présentation des résultats peut prendre des formes diverses : tables rondes, dossiers, expositions etc. Les discussions accompagnant ce processus de travail et les réflexions sur la construction d'un sens historique sont également d'une grande importance. ${ }^{30}$

Une question qui relève de ce genre de travail est celle de la relation entre l'acquisition de connaissances historiques et la capacité de commémoration. ${ }^{31}$ Christian Geißler-Jagodzinski et Verena Haug nous rappellent que les lieux historiques transformés en lieux de commémoration sont en général marqués par une codification culturelle. ${ }^{32}$ Tandis qu'un cimetière est en soi un lieu d'enterrement dans le respect et la dignité, les lieux de commémoration se trouvent, pour la plupart, sur des lieux où ont été commis des crimes contre l'humanité, où des hommes, des femmes et des enfants sont morts sous la torture et dans des conditions atroces, ce sont alors ces victimes qui exigent le respect. Ce respect fait partie d'un «savoir culturel » et il est à apprendre, tout comme l'attitude respectueuse vis-à-vis des victimes. ${ }^{33}$ Depuis un certain temps, il y a d'ailleurs de plus en plus de projets d'art (moderne) dans la conception des lieux de commémoration ${ }^{34}$, même des projets d'art avec des élèves qui ont souvent besoin d'installer des symboles d'espoir dans des lieux marqués autrefois par le désespoir. ${ }^{35}$

28 Ibid., op. cit., p. 320-321.

29 Harald Welzer, "Für eine Modernisierung der Erinnerungs- und Gedenkkultur ", in Gedenkstättenrundbrief 162 (8/2011), p. 3-9.

30 Cf. Ulrich Mayer, op. cit., p. 397-406.

31 Christian Geißler-Jagodzinski et Verena Haug, op. cit., p. 317.

32 Ibid., p. 314.

33 Ibid., p. 314.

34 Cf. par exemple les contributions dans l'ouvrage collectif dirigé par Birgit Dorner et Kestin Engelhardt : Birgit Dorner et Kestin Engelhardt (éd.), Arbeit an Bildern der Erinnerung. Ästhetische Praxis, außerschulische Jugendbildung und Gedenkstättenpädagogik. Stuttgart, Lucius \& Lucius, Dimensionen Sozialer Arbeit und Pflege 9, 2006.

35 Cf. à ce sujet par exemple Iris Groschek, " Der Koffer als Symbol in der Erinnerungskultur. 
On aborde également avec les élèves la question de l'interprétation du passé, l'analyse et la critique de la représentation du passé telle qu'elle est proposée aux visiteurs. ${ }^{36}$ Quand on examine un monument historique - comme par exemple une statue équestre - érigé à une autre époque, ou même une plaque commémorative contemporaine, une des questions qui se posent est celle de la relation entre le site et la représentation collective et/ou régionale du passé. Quel est le rôle du site pour la transmission et la diffusion de cette représentation, pour la transmission d'un certain regard sur le passé ${ }^{37}$ Mais aussi : quelle est l'influence des idées et des valeurs de la société ou d'un certain groupe social sur l'aménagement et l'agencement, sur l'organisation ou bien l'interprétation du lieu $?^{38}$ Les élèves font ainsi une expérience fondamentale, ils comprennent que l'attribution de signification, la construction d'un sens historique se fait également par le "spectateur », par le visiteur, la postérité, et que - dans la plupart des cas - un lieu avait, à une autre époque, une signification différente de celle d'aujourd'hui.

\section{La dimension didactique}

Dans sa dimension épistémologique, la didactique de l'histoire s'intéresse aux modes de transmission de l'histoire dans la société. L'objet principal d'analyse sont les représentations du passé sous leurs formes les plus diverses, considérées comme manifestations de la conscience historique dans la société. La didactique de l'histoire développe des approches analytiques pour étudier des conceptions de l'histoire et met à son tour en avant des concepts d'enseignement, soit pour l'enseignement scolaire, soit pour d'autres contextes sociaux et culturels. Par conséquent, la dimension didactique du débat sur les lieux historiques touche aux représentations du passé liées aux sites historiques ainsi qu'aux constructions de sens historique qu'elles impliquent.

Dans la plupart des cas, les lieux historiques subissent certains aménagements - soit simplement dans un but de sauvegarde et de conservation, de restauration ou d'entretien, soit dans un but de reconstruction, souvent accompagnée d'une information mise à la disposition du grand public. Selon les catégories didactiques, ce genre d'agencement relève de la "culture historique ", qui reflète les valeurs sociales, culturelles et politiques et, pour ainsi dire, une certaine gestion du passé (de toutes les époques) par la société. L'auteur emploie ici le terme de "culture historique " dans la définition que lui a donnée Jacques Le Goff, selon laquelle la culture historique fait partie

Das Beispiel der Gedenkstätte Bullenhuser Damm ", in zeitschrift für didaktik der gesellschaftswissenschaften (zdg) 1/2013, p. 76-99, ici p. 94-95.

36 Cf. Ulrich Mayer, op. cit., p. 401.

37 Ibid., p. 401.

38 Ibid., p. 401. 
du discours sur le " rapport qu'entretient [...] une société avec son passé ». ${ }^{39}$ Les questions liées à la mémoire et à la commémoration font donc également partie intégrante de ce discours. En tant que "représentations matérialisées de la conscience historique $»^{40}$ et manifestations du rapport qu'entretient la société avec son passé, tous les aménagements, tous les agencements, toutes les conceptions d'expositions et les visites guidées comportent forcément des constructions de sens historique. Les indices en sont assez évidents : beaucoup d'agencements de sites obéissent à une certaine dramaturgie. La lumière, l'éclairage et/ou les couleurs d'une salle relèvent d'un symbolique culturellement ancrée ${ }^{41}$ (comme l'opposition de l'obscurité et de la lumière, des ténèbres et de la clarté, dans d'autres lieux les couleurs de l'or, du rouge, etc.). L'aménagement des sites historiques et encore plus celui des lieux de commémoration sont alors marqués par une polarité : d'un côté, les sites permettent une approche émotionnelle du passé, de l'autre côté la visite d'un site apporte également un élément à la compréhension rationnelle et cognitive des événements. ${ }^{42}$ La mise en contexte des objets exposés et des informations dans les centres de documentation est donc d'une grande importance. ${ }^{43}$

39 Jacques Le Goff, Histoire et mémoire, Paris, Gallimard, Folio Histoire, 1988, p. 218-219. Le Goff a repris, de son côté, la notion de "culture historique » employée par Bernard Guenée dans Histoire et culture historique dans l'Occident médiéval (1980). B. Guenée rassemblait sous ce terme d'une part le "bagage professionnel " des historiens, de l'autre " le public et l'audience des historiens ». Le Goff, op. cit., p. 218. Dans le discours allemand, la notion de " culture historique " a été développée par exemple par Jörn Rüsen, Bernd Schönemann, Wolfgang Hardtwig et d'autres auteurs.

40 La formule originale employée par Jörn Rüsen est la suivante : "Geschichtskultur ist die praktisch wirksame Artikulation von Geschichtsbewusstsein im Leben einer Gesellschaft ». Jörn Rüsen, "Was ist Geschichtskultur? Überlegungen zu einer neuen Art, über Geschichte nachzudenken », in Klaus Füßmann, Heinrich Theodor Grütter et Jörn Rüsen (éd.), Historische Faszination. Geschichtskultur heute, Köln/Weimar/Wien, Böhlau, 1994, p. 3-26, ici p. 5. La nécessité d'un élargissement du concept de J. Rüsen a été postulée par Bernd Schönemann. Bernd Schönemann, "Geschichtsdidaktik und Geschichtskultur ", in Bernd Mütter, Bernd Schönemann et Uwe Uffelmann (éd.), Geschichtskultur. Theorie - Empirie - Pragmatik, Weinheim, Schriften zur Geschichtsdidaktik 11, 2000, p. 26-58.

41 Dans un article récent, Iris Groschek réfléchit à cette dimension des lieux de commémoration. L'exemple analysé par I. Groschek est le mémorial de la déportation "Bullenhuser Damm ». Iris Groschek, op. cit., ici p. 90-91. On trouvera dans cet article de plus amples références bibliographiques sur ce sujet.

42 Phil. C. Langer, «Fünf Thesen zum schulischen Besuch von KZ-Gedenkstätten », in Einsichten und Perspektiven. Themenheft Nr.1 Holocaust Education, München, 2008, p. 66-75, cité ici selon Iris Groschek, op. cit., ici p. 90.

43 Cf. par exemple Stefanie Schüler-Springorum, "Nationale Schande, Universelle Lehre. Die Darstellung von Nationalsozialismus und Holocaust in Museen ", in Gerhardt Paul et Bernhard Schoßig (éd.), Öffentliche Erinnerung und Medialisierung des Nationalsozialismus. Eine Bilanz der letzten dreißig Jahre, Göttingen, 2010, p. 138-153 (ici p. 139), cité selon Iris Groschek, op. cit., ici p. 91-92. 
Très souvent, la discussion sur les arrangements pédagogiques sur les sites mêmes, adressés à un public plus large, implique un débat sur la gestion de la mémoire. ${ }^{44}$ Bien que cette question touche en principe tous les lieux historiques aménagés pour des visiteurs, ce sont surtout les lieux de commémoration dont l'agencement et la conception pédagogiques sont discutés dans le débat sur la mémoire collective et sociale, sur la fonction de la mémoire dans la société ainsi que dans celui sur la commémoration dans l'ensemble de la société et au niveau politique. ${ }^{45}$ Depuis un certain temps, l'historique de la conception d'un lieu de commémoration est également évoqué et rendu transparent pour le public, ce qui donne au site une "mémoire " propre et, selon Peter Reichel, ce qui lui enlève en même temps une certaine "valeur suggestive » et " authentique ». ${ }^{46}$

Une autre question discutée régulièrement ${ }^{47}$ est la suivante : qu'est-ce que les visiteurs retiennent lors d'une visite des lieux de commémoration ? N'y aurat-il pas par exemple le risque d'une "glorification " (quoique involontaire) des crimes par un centre de documentation ? C'est une question posée par exemple par Peter Reichel, professeur en sciences politiques à l'Université de Hambourg. Pourquoi les gens vont-ils visiter des anciens lieux de crimes? P. Reichel a voulu étudier et comprendre la fonction de ces lieux pour les visiteurs. Dans un article qu'il consacre aux raisons pour lesquelles la représentation publique du régime national-socialiste est une discussion sans fin en Allemagne, P. Reichel met en relief la complémentarité des lieux de crimes et des lieux présentés comme lieux d'attraction (comme par exemple les colonies de vacances créées par l'organisation nationale-socialiste Kraft durch Freude [" la force par la joie »]). Selon lui, c'est uniquement la prise en compte de cette complémentarité qui rend possible

44 Ulrich Haase, "Rede zur Eröffnung des Symposiums ", in Burkhard Asmuss et HansMartin Hinz (éd.), Historische Stätten aus der Zeit des Nationalsozialismus. Orte des Erinnerns, des Gedenkens und der kulturellen Weiterbildung? Zum Umgang mit Gedenkorten nationaler Bedeutung in der Bundesrepublik Deutschland. Symposium am 23. und 24. November 1998, im Deutschen Historischen Museum, Berlin, Deutsches Historisches Museum, 1999, p. 13-20.

45 Cf. par exemple Peter Reichel, "Die umstrittene Erinnerung. Über Ursachen der anhaltenden Auseinandersetzung um die öffentliche Darstellung der NS-Vergangenheit ", in Burkhard Asmuss et Hans-Martin Hinz (éd.), op. cit., p. 21-37, ici p. 22-23, p. 29 ; Jan Philipp Reemtsma, "Wozu Gedenkstätten? ", in Aus Politik und Zeitgeschichte (APuZ), 25-26/2010, p. 3-9 ; Volkhard Knigge, op. cit., p. 10-16 ; Harald Welzer, «Erinnerungskultur und Zukunftsgedächtnis ", in Aus Politik und Zeitgeschichte (APuZ), 25-26/2010, p. 16-23 ; Harald Welzer, "Für eine Modernisierung der Erinnerungs- und Gedenkkultur ", in Gedenkstättenrundbrief 162 (8/2011), p. 3-9.

46 Peter Reichel, op. cit., ici p. 31.

47 Cf. par exemple le compte rendu du colloque " Gedenkstätten und Geschichtspolitik " (qui s'est tenu du 31 mai au $1^{\text {er }}$ juin 2013 à Fürstenberg/ Havel, organisé par la KZ Gedenkstätte Neuengamme et la Mahn- und Gedenkstätte Ravensbrück). Jutta Mühlenberg (Hamburg), "Gedenkstätten und Geschichtspolitik ", compte rendu du colloque (en ligne) : http:// hsozkult.geschichte.hu-berlin.de/tagungsberichte/id=4890. 
une vue d'ensemble du régime. ${ }^{48} \mathrm{P}$. Reichel souligne que les lieux de commémoration nous rappellent les victimes, ou plus précisément les crimes et les victimes, et qu'ils mettent en évidence un lien compliqué entre la barbarie et la civilisation..$^{49} \mathrm{Il}$ suppose que l'intérêt principal du public lors d'une visite est de se rappeller la résistance intérieure et extérieure ainsi que l'exil des opposants, et de reconnaitre à travers cette mémoire ses devoirs, ses obligations et ses responsabilités pour le futur. P. Reichel pense que les visiteurs se rendent dans des lieux de commémoration afin de se rappeller les principes anti-totalitaires qui représentent - d'un point de vue politique - les fondements de la République fédérale d'Allemagne. ${ }^{50}$

Or, depuis les années 1990, un certain nombre de lieux historiques jusqu'alors peu considérés commencent à faire surface dans la perception publique - les " lieux liées aux acteurs du régime " qui pendant un certain temps ont fait l'objet d'un "tourisme historique sauvage $»^{51}$. Ainsi, vers la fin des années 1970, l'ancien «terrain de la Gestapo " à Berlin fut visité par des touristes et des scolaires. Le débat public qui en résulta au cours des années 1980 aboutit à la décision d'y installer un centre de documentation, la « Topographie de la terreur ", et de laisser en friche la plus grande partie du terrain ${ }^{52}$. De même, la ville de Nuremberg, après avoir constaté l'intérêt public croissant pour le terrain des grands rassemblements du parti national-socialiste (là aussi surtout de la part des jeunes et des touristes, depuis les années 1970), décida d'y installer un centre de documentation - décision et construction, très controversées, accompagnées par un vif débat public, politique et scientifique. ${ }^{53}$ De pareils débats ont été menés autour d'autres lieux semblables. Depuis quelques années, le débat

48 Peter Reichel, op. cit., p. 21.

49 Ibid., p. 29 ; cf. aussi Jan Philipp Reemtsma, op. cit. Se référant à James E. Young, un judaïste américain, Peter Reichel souligne que la visualisation des événements horribles et leur représentation par des objets (par exemple les vêtements, lunettes et autres objets personnels des déportés) représente toujours une reconstruction, le plus souvent liée à un appel moral. Peter Reichel, op. cit., p. 28. Quant aux acteurs, ils sont pour ainsi dire " présents » dans des bâtiments publics toujours existants, lieux qui font toujours objet de discussion, mais qui, selon Reichel, sont indispensables pour rendre évidente l'interdépendance entre un mouvement de masse, la fascination pour un «Führer » et des crimes épouvantables. Peter Reichel, op. cit., p. 29.

50 Peter Reichel, op. cit., p. 23.

51 «Ein ganz ungeregelter historischer Tourismus » : Christoph Stölzl, "Begrüßung », in Burkhard Asmuss et Hans-Martin Hinz (éd.), op. cit., p. 11-12.

52 Cf. Gabriele Camphausen, "Internationales Dokumentations- und Begegnungszentrum Topographie des Terrors, Berlin ", in Burkhard Asmuss et Hans-Martin Hinz (éd.), op. cit., p. 165-174. Ce lieu historique a été transformé en friche pendant un certain temps. Camphausen considère le débat public et le changement de perception comme une prise de conscience de la société. Ibid., p. 167.

53 Cf. Franz Sonnenberger, « Eine Stadt stellt sich ihrer Vergangenheit. Dokumentationszentrum Reichsparteitagsgelände Nürnberg ", in Burkhard Asmuss et Hans-Martin Hinz (éd.), op. cit., p. 45-67. Cf. aussi les publications de Harald Welzer, Volkhard Knigge, Christian GeißlerJagodzinski et Verena Haug, Jan Philipp Reemtsma et d'autres cités dans cet article. 
sur la gestion des lieux de commémoration et des lieux liés aux acteurs fait donc partie intégrante du débat plus large sur "l'avenir de la mémoire " ${ }^{54}$ (Zukunft der Erinnerung) en Allemagne.

La didactique de l'histoire fait l'analyse des interdépendances dans ces débats, de la "politique de la mémoire " et des mécanismes sociaux de la mémoire d'un côté, et l'analyse des agencements des sites de l'autre. Par effet d'engrenage, tous ces aspects ont un impact important sur la représentation de l'histoire dans la société.

\section{La dimension politique, sociale et culturelle}

Les aspects mentionnés à la fin des paragraphes précédents nous amènent déjà au cour de la troisième composante du débat allemand sur les lieux historiques, la dimension politique, sociale et culturelle. Elle comprend les questions d'agencement d'un lieu ou d'un site - ruine ou bâtiment à entretenir, musée, lieu de commémoration etc. - abordées dans l'espace public ainsi que la discussion sur la fonction sociétale des monuments. ${ }^{55}$ Outre les questions financières qui se posent régulièrement dans ce contexte, les discussions menées par les communes, les conseils municipaux ou les associations - souvent reprises par les médias et un public plus large - tournent autour des causes et des objectifs liés à la sauvegarde ou à l'aménagement d'un lieu historique, visant par exemple la culture générale ou l'éducation à la citoyenneté. Beaucoup de communes s'engagent à rendre des lieux historiques régionaux accessibles afin de faciliter la découverte de l'histoire locale et régionale et afin de la situer par rapport à l'histoire mondiale ou du moins européenne. Très souvent, il s'agit aussi de l'engagement de bénévoles et d'associations fondées dans ce but. En Hesse par exemple, il y a un bon nombre de "Cercles historiques " qui s'engagent à sauvegarder le patrimoine des huguenots immigrés dans la région au XVII et XVIII ${ }^{\mathrm{e}}$ siècles $^{56}$ ou celui du patrimoine historique de la vie rurale.

Au cours des dernières décennies, plusieurs points qui ont évolué peuvent être observés quant à ces activités. Pendant les années 1970, incitées par le mouvement des " ateliers d'histoire ", de l'histoire "vu d'en bas " et par une nouvelle approche de la recherche historique fondée sur les témoignages oraux (oral history), beaucoup d'activités et de réflexions portaient sur l'histoire locale ou régionale, et concernaient par exemple l'histoire d'une entreprise, d'une industrie régionale ou d'un atelier spécifique. Les évolutions dans le monde du travail et dans l'économie ont ainsi entraîné la création de nombreux musées sur la vie industrielle d'autrefois.

54 Volkhard Knigge, op. cit., p. 10-16.

55 Peter Reichel, op. cit., p. 29-30.

56 Par exemple les sites en ligne : www.hugenotten-waldenserpfad.eu; www.hugenottenmuseum. de ; www.kelze-online.de. 
Depuis les années 1970, on peut également constater un engagement civique croissant pour une analyse critique de la période du national-socialisme. Par la suite, le changement dans la perception des lieux depuis la fin des années 1970 et au cours des années 1980 a abouti à une réflexion sur une conception didactique et pédagogique, par exemple sous la forme d'expositions, de débats publics, etc. ${ }^{57}$ Les années 1980 ont vu en effet un essor de projets de recherche locale (entre autres avec des élèves) aboutissant à des expositions locales ou régionales sur l'implication d'une ville ou d'une région dans le régime nazi. ${ }^{58}$ Les changements dans la conception des lieux de commémoration se poursuivit pendant les années 1990, c'est-à-dire après l'unification de l'Allemagne. Selon la théorie de Jan Assmann sur la transition de la "mémoire par la communication " à la " mémoire culturellement transmise " ${ }^{59}$, moment situé environ quarante ans après un événement, les années 1980 représenteraient ce tournant d'un mode mémoriel vers un autre. Selon C. Geißler-Jagodzinski et V. Haug, la " culture de la mémoire » de ces années-là implique dans sa dimension politique un message adressé aux voisins : l'Allemagne ne reprendra pas la politique de pouvoir mené au début du $\mathrm{XX}^{\mathrm{e}}$ siècle. ${ }^{60}$ L'engagement pour un agencement des lieux de commémoration et l'enseignement historique sur ces lieux « extrascolaires " se font ainsi sous le signe de la commémoration des victimes. ${ }^{61}$

La discussion portant sur les sites de la période national-socialiste, surtout sur les anciens camps de concentration transformés en lieux de commémoration, est spécifique au sein du volet politique du débat sur les lieux historiques. ${ }^{62}$ Depuis 2005, les lieux de commémoration peuvent obtenir des subventions publiques à condition d'avoir élaboré un concept pédagogique ${ }^{63}$ pour la mise en place d'une exposition, pour des visites scolaires, des groupes ou des visites individuelles. Selon Jan Philipp Reemtsma, directeur administratif de l'Institut de recherche en Sciences Sociales à Hambourg (Hamburger Institut

57 Cf. aussi Christian Geißler-Jagodzinski et Verena Haug, op. cit. À propos d'une histoire de la gestion des anciens bâtiments publics du régime national-socialiste (à commencer par la destruction de certains bâtiments, la réutilisation et jusqu’à la transformation en lieu de commémoration) depuis 1945 jusqu’en 2008 ; cf. Winfried Nerdinger, « Die Dauer der Steine und das Gedächtnis der Architekten », in Peter Reichel et al. (éd.), op. cit., p. 378- 397.

58 Christian Geißler-Jagodzinski et Verena Haug, op. cit., p. 305.

59 Jan Assmann, Das kulturelle Gedächtnis. Schrift, Erinnerung und politische Identität in antiken Hochkulturen, München, Beck Verlag, 2007. Jan Assmann a developpé sa théorie sur la «mémoire par la communication» et la « mémoire culturellement transmise » en élargissant la théorie de Maurice Halbwachs sur la "mémoire collective " d'un côté et en s'appuyant sur ses propres recherches sur l'Égypte ancienne de l'autre.

60 Christian Geißler-Jagodzinski et Verena Haug, op. cit., p. 308.

61 Ibid., p. 305.

62 Peter Reichel, op. cit., p. 22-23.

63 Cf. Christian Geißler-Jagodzinski et Verena Haug, op. cit., p. 310 ; cf. aussi Deutscher Bundestag, Drucksache 16/9875 vom 19.06.2008, Fortschreibung der Gedenkstättenkonzeption des Bundes. 
für Sozialfoschung), ces lieux de commémoration sont les lieux d'une prise de conscience quant à la " fragilité de notre civilisation ». ${ }^{64} \mathrm{~J}$.-P. Reemtsma souligne le fait que ces lieux nous rappellent que notre société, mais aussi la démocratie et la civilisation sont constamment menacées, et que le processus de civilisation n'est pas irréversible. ${ }^{65}$ Christian Geißler-Jagodzinski et Verena Haug mettent l'accent sur la part d'incompréhensible, d'inconcevable, liée à l'extermination " industrielle » de vies humaines sous le régime national-socialiste. ${ }^{66}$

L'organisation des anciens camps de concentration en lieux de commémoration comporte donc une problématique spécifique et a aussi son propre historique. Au début, dans l'immédiat après-guerre, ce sont en majorité des associations d'anciens déportés qui s'en sont occupé. ${ }^{67}$ Ainsi, pendant les années 1960, les premiers lieux de commémoration furent les anciens camps de concentration de Dachau, Flossenbürg et Bergen-Belsen. On pouvait y trouver des petites documentations, mais aucune " équipe pédagogique " n'était sur place. En 1965, un mémorial fut installé à Neuengamme, sans documentation spécifique. ${ }^{68}$ Ce qui était important à cette époque, c'était de rendre publics les meurtres et la terreur, de montrer que ces horreurs avaient réellement eu lieu. ${ }^{69} \mathrm{Il}$ y avait donc une sorte d'enseignement de l'histoire pour le grand public, mais les lieux mêmes restaient des lieux de commémoration pour les victimes et leurs proches. ${ }^{70}$ Depuis un certains temps, d'autres groupes se sont impliqués dans la conception des lieux de commémoration : des muséologues, des historiens, mais surtout des enseignants et des pédagogues, ce qui a entraîné des changements dans la conception et l'agencement. Le résultat en est une séparation plus évidente entre les lieux de commémoration mêmes et les musées sur place (de plus en plus médialisés), ce qui enlève à l'ensemble des sites le caractère d'un lieu qui se prête à l'identification biographique. $^{71}$ Le problème du débat politique et pédagogique depuis les années 1970 est donc l'enchevêtrement des questions de mémoire et de commémoration dans la société, des questions de gestion et d'agencement des lieux

64 Jan Philipp Reemtsma, op. cit., p. 9.

65 Ibid., p. 9.

66 Christian Geißler-Jagodzinski et Verena Haug, op. cit., p. 306-307. Les auteurs craignent que la notion de « responsabilité historique pour le futur " mentionnée très souvent dans ce débat ne cache la part d'incompréhensible et d'inconcevable, liée à l'extermination « industrielle » de vies humaines.

67 Cf. aussi Christian Geißler-Jagodzinski et Verena Haug, op. cit., p. 302-303. À propos d'une histoire du débat, de l'installation et des différentes conceptions des lieux de commémoration cf. Stefanie Endlich, «Orte des Erinnerns - Mahnmale und Gedenkstätten », in Peter Reichel et al. (éd.), op. cit., p. 350-377.

68 Christian Geißler-Jagodzinski et Verena Haug, op. cit., p. 303.

69 Ibid., op. cit., p. 303.

70 Ibid., op. cit., p. 304.

71 Peter Reichel, op. cit., p. 30-31. 
ainsi que des questions liées à la "mission pédagogique " des sites. Ainsi, lors d'un colloque en 1998, Ulrich Haase, alors représentant du ministère pour la culture et la recherche, a formulé très clairement les objectifs du travail pédagogique des sites de commémoration selon le ministère : " Il s'agit d'ancrer le souvenir, la mémoire et la commémoration dans la vie quotidienne, comme une partie de la vie et de l'autonomie. Il faut sensibiliser et rester sensible afin d'appréhender toute atteinte portée à la démocratie et à la liberté ". ${ }^{72}$

C'est dans ce sens-là que Christian Geißler-Jagodzinski et Verena Haug, en 2009, critiquent d'une certaine façon le fait que les lieux de commémoration aient abandonné, durant les années 1990, leurs exigences en matière de travail critique concernant la société actuelle, ${ }^{73}$ fonction pourtant d'importance primordiale selon ces deux auteurs. Lors d'un colloque à Fürstenberg en mai 2013, les intervenants et discutants ont souligné à nouveau l'actualité de ce discours, étant donné surtout qu'il y a de plus en plus de groupes intéressés et d'acteurs différents dans le champ de la " politique de l'histoire » et de la " politique de la mémoire $»^{74}$ qui se réferent à des positions parfois controversées.

En résumé, la dimension politique, sociale et culturelle du débat sur les lieux historiques fait partie intégrante du discours sur la mémoire, ${ }^{75}$ sur la " gestion du passé ", la responsabilité éthique et politique et sur les représentations du passé dans la société.

\section{Le travail pédagogique sur les lieux de commémoration}

Le quatrième volet du débat sur les lieux historiques est très spécifique. La transformation des anciens camps de concentration et d'autres lieux liés aux crimes du régime national-socialiste en lieux de commémoration ${ }^{76}$ a engendré un domaine didactique et un champ professionnel très spécifiques, celui des spécialistes de la "pédagogie appliquée aux sites historiques aménagés en lieux de commémoration " (Gedenkstättenpädagogik). La tâche de celle-ci ne se limite pas à une sensibilisation éthique et à un accompagnement des visi-

72 Ulrich Haase, op. cit., p. 18.

73 Christian Geißler-Jagodzinski et Verena Haug, op. cit., p. 309.

74 Cf. Jutta Mühlenberg, op. cit. (compte rendu " Gedenkstätten und Geschichtspolitik »).

75 Les publications à ce sujet sont innombrables et en France et en Allemagne. Citons cependant les noms de quelques auteurs de référence qui ont sensiblement influencé les approches analytiques : Maurice Halbwachs, Pierre Nora, Jacques Le Goff, Michel de Certeau, Jan Assmann, Aleida Assmann, Jörn Rüsen. Quant au sujet de la «culture de la mémoire » en Allemagne depuis les années 1990, cf. par exemple Bernd Faulenbach, "Öffentliches Erinnern im vereinten Deutschland und in Osteuropa seit den 1990er Jahren ", in Petra Haustein et al. (éd.), op. cit., p. 233-249.

76 Quant aux agencements des anciens camps de concentration, camps d'internement, prisons, lieux de travail forcé en RFA et RDA, cf. également Vojtech Blodig, "Die Gedenkstätte Theresienstadt gestern und heute ", in Jürgen Danyel (éd.), op. cit., p. 235-243. 
teurs, il s'agit aussi d'une mise à disposition des lieux afin de rendre ceux-ci " accessibles" selon les exigences des connaissances scientifiques. D’après les catégories de la didactique de l'histoire, ce travail sur les lieux de commémoration fait partie intégrante de la "culture historique $» .^{77}$

Or, selon Christian Geißler-Jagodzinski et Verena Haug, ${ }^{78}$ les fonctions des centres de documentation installés sur les lieux de commémoration sont tout d'abord l'information et l'enseignement historique ${ }^{79}$, dans le but de rendre l'empathie envers les victimes possible ${ }^{80}$ et, de manière générale, une "éducation civique pour la démocratie " ${ }^{81}$. Lacquisition de connaissances historiques est primordiale selon ces deux auteurs, car la connaissance des structures, des mécanismes et des motivations qui ont mené aux crimes nazis ${ }^{82}$ peut donner aux élèves des critères pour analyser les évolutions politiques contemporaines de façon critique. ${ }^{83}$

De plus, C. Geißler-Jagodzinski et V. Haug posent la question de savoir si le travail pédagogique effectué sur les lieux de commémoration implique une fonction critique vis-à-vis de la société, ce qui impliquerait une analyse du déve-

77 Cf. Jacques Le Goff, op. cit., p. 218-19. À propos du discours sur la mémoire, cf. par exemple Günter Morsch, «Von Denkmälern und Denkmalen. Von Gedenkstätten und Zeithistorischen Museen », in Jürgen Danyel (éd.), op. cit., p. 181-186 ; Gudrun Schwarz, "Zur Gedenkstätte Ravensbrück ", in Jürgen Danyel (éd.), op. cit., p. 153-162 ; Jürgen Zarusky, "Die KZ-Gedenkstätte Dachau: Anmerkungen zur Geschichte eines umstrittenen historischen Ortes », in Jürgen Danyel (éd.), op. cit., p. 187-196 ; Moshe Zimmermann, « Die Erinnerung an Nationalsozialismus und Widerstand im Spannungsfeld deutscher Zweistaatlichkeit ", in Jürgen Danyel (éd.), op. cit., p. 133-138 ; Peter Reichel, Harald Schmid et Peter Steinbach, " Nach dem Ende nationaler Nachkriegsmythen - eine europäische Erinnerungskultur? ", in Peter Reichel et al. (éd.), op. cit., p. 398-415.

78 Christian Geißler-Jagodzinski et Verena Haug, op. cit., p. 311 et suivantes.

79 Iris Groschek souligne même que les destinataires de cet enseignement sont surtout les gens qui n'ont pas la possibilité de s'entretenir directement avec des témoins. Cf. Iris Groschek, op. cit.

80 Christian Geißler-Jagodzinski et Verena Haug, op. cit., p. 315-316.

81 Ibid., p. 309.

82 Les auteurs mettent, dans ce contexte, en relief les attitudes de cupidité, d'ignorance, d'indifférence, d'obéissance aveugle, la prétention à appartenir à une caste supérieure, le mépris de l'homme et de la vie humaine, et le rascisme. Christian Geißler-Jagodzinski et Verena Haug, op. cit., p. 313.

83 Christian Geißler-Jagodzinski et Verena Haug, op. cit., p. 325. Quant au discours pédagogique et didactique concernant l'éducation civique pour la démocratie (ou éducation à la citoyenneté), il existe un débat spécifique concernant la place de l'acquisition de connaissances historiques sur le régime national-socialiste par rapport à d'autres sujets historiques. Cf. par exemple "Menschenrechtsbildung, Holocaust Education, Demokratieerziehung ", in Zeitschrift für Geschichtsdidaktik 2012, 11. Jahrgang, Göttingen, 2012 ; Bodo von Borries, Geschichtslernen und Menschenrechtsbildung. Auswege aus einem Missverhältnis? Normative Überlegungen und praktische Beispiele, Wochenschau Verlag, Schwalbach/Ts., 2011 ; Peter Henkenborg, "Politische Urteilsfähigkeit als politische Kompetenz in der Demokratie - der Dreiklang von Analysieren, Urteilen und Handeln ", in zeitschrift für didaktik der gesellschaftswissenschaften, Heft 2/2012, p. 28-50. 
loppement historique et une mise en relief des possibilités qui existe pour la participation civique, sociétale et politique de tout un chacun. ${ }^{84}$ Cette conception du travail pédagogique comme travail " critique " allant vers le développement de la démocratie vient des années 1970 et 1980, lorsque les initiatives qui cherchaient à ériger des lieux de commémoration se voyaient obligées de " déblayer" (au sens propre et au figuré : parfois des ronces, parfois d'autres utilisations pragmatiques quelconques) les ancien lieux de détention, de torture et de meurtre, mais aussi les lieux de la vie juive en Allemagne. On comprend alors qu'ils aient considéré leur travail comme un engagement critique. ${ }^{85}$ Quant au développement de l'empathie envers les victimes, C. Geißler-Jagodzinski et V. Haug soulignent que les lieux de commémoration sont fortement marqués par une codification culturelle : il faut apprendre le respect et une attitude respectueuse vis-à-vis des victimes, comportement qui ne semble pas aller de soi pour un certain nombre de visiteurs. ${ }^{86}$ Une autre dimension de ce travail pédagogique tend à développer les capacités politiques et morales des élèves en mettant en relief l'impact du passé et de l'histoire sur le présent, la signification de l'histoire pour l'interprétation du présent et pour la projection du futur. ${ }^{87}$

\section{Conclusion}

Afin d'illustrer la complexité du sujet, l'auteur a choisi de présenter dans cet article quatre composantes du débat allemand sur les lieux historiques. Sur le volet pédagogique, les réflexions scientifiques et la méthodologie de l'enseignement portent sur l'apprentissage des élèves par le biais d'une méthode de travail sur place, sur l'acquisition de connaissances historiques et de compétences historiennes et sur l'élaboration de représentations qui sont les plus proches possibles des connaissances scientifiques actuelles. Cependant, le débat sur les lieux historiques touche non seulement aux domaines de la recherche, de l'enseignement et des connaissances historiques, mais aussi un large public. L'impact (supposé) des lieux historiques sur les identités régionales engendre une dimension politique, sociale et culturelle qui touche d'un côté la sauvegarde du patrimoine et - aspect très important -, de l'autre côté, les lieux de commémoration. Par conséquent, ce volet du débat implique une réflexion et une discussion sur la fonction qu'occupe la mémoire dans la société, et souligne l'importance de la transmission des valeurs démocratiques aux générations futures.

84 Christian Geißler-Jagodzinski et Verena Haug, op. cit., p. 299-302.

85 Ibid., p. 301.

86 Ibid., p. 314.

87 Ibid., p. 320-321. Christian Geißler-Jagodzinski et Verena Haug mettent néanmoins en avant qu'il peut y avoir des contradictions entre les objectifs adoptés par des lieux de commémoration mêmes et les objectifs imposés par la société. Ibid., p. 301. 
La " pédagogie appliquée aux sites historiques aménagés en lieux de commémoration " (Gedenkstättenpädagogik) représente un domaine très spécifique dans le cadre des réflexions pédagogiques et du débat politique, social et culturel.

Toutes ces dimensions comprennent également un volet didactique épistémologique, quasiment inséparable des questions abordées, mais qui a néanmoins été présentée ici dans une approche analytique. La didactique de l'histoire analyse le discours politique, social et culturel et surtout la « culture historique " - par exemple les choix qui ont présidé à l'agencement d'un lieu ou des aménagements liés à "l'enseignement" - par laquelle s'exprime la relation que la société entretient avec son passé. Sur le volet didactique, on trouve donc le travail de réflexion et d'analyse concernant l'acquisition des connaissances historiques ainsi que l'analyse des mécanismes par lesquels s'effectue la transmission des idées et des représentations du passé.

Christine Pflüger

Université de Kassel christine.pflueger@uni-kassel.de

\title{
Résumé
}

Cet article présente les questions qui animent le débat allemand sur les lieux historiques et leur valeur pédagogique. Dans la recherche historique, les sites de tout ordre (aménagés ou non) sont considérés comme des sources historiques ayant une valeur épistémologique propre, qui favorise l'acquisition de connaissances historiques et influence l'élaboration de représentations de l'histoire. Les anciens camps de concentration du régime national-socialiste en Allemagne qui ont été transformés en lieux de commémoration constituent une catégorie spécifique de lieux historiques, suscitant un débat sur la mémoire dans la société.

\section{Mots-clés}

Lieux historiques, lieux de commémoration, enseignement de l'histoire, mémoire, Allemagne.

\begin{abstract}
Being important sources for the historian, historical places, sites, monuments and landscapes may also help students in developing historical knowledge, concepts and representations of history. This article deals with the German didactic theory on learning and teaching history by going out of the classroom and working on historical sites. Within this debate, memorials are considered as a special kind of historical places, taking an important place in public and scientific debate about memory, society and heritage education and as a topic in the didactics of history.
\end{abstract}

\section{Keywords}

Historic sites, commemoration sites, teaching history, memory, Germany. 